\title{
A comparison of three geostatistical procedures for rainfall network optimization
}

\author{
HAIFA FEKI ${ }^{1}$ \& MOHAMED SLIMANI ${ }^{2}$ \\ 1 Higher School of Engineers of Rural Equipment of Medjez el beb, Tunisia \\ haifa.fki@gmail.com \\ 2 National institute of agronomy of Tunisia
}

\section{INTRODUCTION}

A network of monitoring stations is designed to give the most possible representative picture of a meteorological field state which varies continuously in time and space. As the cost of installing and maintaining a network of observation stations is very high, an optimal monitoring network should be sought. The optimization of the network is, therefore, necessary and essential to identify gaps in the network and eliminate any redundancy. The methodology consists of a sequence of steps which provides different information about the distribution. The major steps are the following: compiling a valid database, monitoring network analysis, structural analysis including variography, and interpolation using geostatistical predictors. In addition, other variables may be introduced as supplementary information to ameliorate these maps. The purpose of this article is to apply and compare three geostatistical multivariate techniques (kriging with external drift, regression-kriging and cokriging) which allow the introduction of a second variable, in order to estimate the monthly average rainfall and to present their related error maps. Therefore, regions where the estimation error is important are represented by adding new stations.

\section{CASE STUDY}

Tunisia is a Mediterranean coastal region placed on the north of Africa with an area of $164150 \mathrm{~km}^{2}$. The physical geography of the region is quite heterogeneous. Climatologically, Tunisia is situated in the geographic transition zone between humid temperate climate and the arid Saharan climate. A total of 140 rainfall stations of the Institut National de la Météorologie (INM) and the Direction Générale des Ressources en Eau (DGRE) networks are used. For all these stations, the monthly rainfall means are available for a period varying from 20 to 100 complete years, from 1901 to 2000 .

\section{METHODOLOGY}

In geostatistics, when the variable of interest is sparse or poorly correlated in space, the prediction of this variable over the whole study area may be improved by accounting for secondary information exhaustively sampled over the same study area. The secondary information can be incorporated using kriging with external drift, regression-kriging, and cokriging. The autocorrelation between datasets is described by the semi-variogram, which is one of the significant functions to indicate spatial correlation in observations measured at sample locations (Slimani et $a l$. 2011). The optimization method applied in this study is to use the results of previous interpolation systems that provide, in addition to the interpolated values, an estimate of the error. Its reduction is the most used criterion for the optimization of the rainfall network (Feki 2010).

\section{RESULTS AND DISCUSSION}

The power model is the most presented one, which indicates the presence of a drift. Fluctuations are present around the drift and are due to the presence of an orographic barrier. The preceding results are explained by the fact that, during the dry season; rainfall is of convective type, therefore in direct relation with the relief. This is in contrast to the winter, when the disruptions reach thousands of kilometers, rainfall is indifferent to the orographic obstacles. 
For the kriging with external drift and regression-kriging, a digital elevation model at $20 \times$ $20 \mathrm{~m}$ resolution was used. The precipitation estimates by kriging with external drift show that during the rainy months the pattern of precipitation contours take the aspect of the bends of the DEM in the regions with very weak densities of stations: these are principally the regions of the south - where one can recognize the chain of the Dhahar, and the high altitudes of the Dorsale.

Examination of the iso-deviations maps has allowed us to quickly locate the parts of a region where the standard deviation of the error estimation is the weakest, as well as those where they are the highest. Logically, any part of a region where the interpolation standard deviation is high is subject to an increased density of stations. The central and southern regions of the country represent the highest values of variance estimation. Thus, these regions are considered for the implementation of new stations; however, we have chosen to place 11 stations 'as fictitious points' in all the regions of the country, in order to have a better view of the kriging variance reduction. The information gain at any point of the study area is then typically assessed in terms of reduction of the kriging variance: this type of approach is therefore an analysis of variance reduction (Feki, 2010). The mapping of this indicator (gain) shows that the maxima are in the direct vicinity of the new stations, reaching $40 \%$ in the south zone (for the month of May), and vary between 20 and $25 \%$ in the centre, unlike the north where the maximum precision obtained is only $10 \%$.

We will qualify the distance over which the gain is strictly positive in the neighbourhood of a new installation in a given region as the average radius of influence. By comparing this radius to the range of the secondary structure of omni directional variograms, we can conclude on the sensitivity of the kriging system when adding other information. Indeed, if the average radius of influence goes beyond the range of the secondary structure (variogram with hole effect), the interpolation system will certainly be sensitive to the addition of further information. As the experimental omni directional variograms include all types of nesting, they consequently present secondary structures in which we can determine the lower range. In our case, we can confirm that in the north of the country, the interpolation system is not sensitive to adding further information, so, the existing network describes, in a satisfactory way, the spatial variability of the rainfall. In the centre of the country, it is moderately sensitive to the addition of new stations regardless of the season. In contrast, in the south, the network is insufficient to represent the spatial variability of rainfall, especially in autumn.

\section{CONCLUSION}

This paper has presented three geostatistical algorithms with elevation as an auxiliary variable (kriging with external drift, regression-kriging and cokriging) for predicting monthly averaged rainfall. We are interested in optimizing the rainfall network in Tunisia based on reducing the variance of kriging. For this we have drawn maps of deviations of estimation errors to locate areas with high values of standard deviations. Then, we conducted a test implementation of some new stations throughout the country and assessed the gain. We have concluded that in northern Tunisia the system interpolation is not sensitive when adding additional information, and therefore the existing network satisfactorily describes spatial variability of rainfall; indeed the maximum gain precision obtained does not exceed $10 \%$. In central Tunisia the system is averagely sensitive to adding new stations, irrespective of season; the gain precision varies between 20 and $25 \%$. By contrast, in the south the gain accuracy can reach $40 \%$ indicating that the network is insufficient to represent the spatial variability of rain, especially in autumn.

\section{REFERENCES}

Feki, H. (2010) Pour une optimization régionalisée du réseau des stations météorologiques: interpolation géostatistique à pas de temps mensuel et annuel. Mémoire de these de doctorat en sciences agronomiques, INAT, Tunisie.

Slimani. M. Cudennec. Ch. \& Feki. H. (2011) Structure du gradient pluviométrique de la transition Méditerranée-Sahara en Tunisie: determinants géographiques et saisonnalité. Hydrological Sciences Journal 52(6), 1088-1102. 